\title{
Écrire de/au-delà de la marge : l'homosexualité et son rapport aux foules dans l'œuvre d'Abdellah Taïa
}

\author{
Thomas Muzart \\ The City University of New York
}

Reconnu comme l'un des premiers auteurs ouvertement homosexuels au Maroc' ${ }^{1}$ Abdellah Taïa a souvent fait part du

\footnotetext{
${ }^{1}$ Si Rachid 0. est reconnu comme le premier à avoir révélé son homosexualité dès 1994 avec L'Enfant ébloui (Boukhari, 2007), Taïa est le premier à publier avec son nom complet, sans se cacher derrière un diminutif ou un pseudonyme. Mis à part cette distinction, Najib Redouane les place tous deux dans la génération d'auteurs contemporains qui, par leur écriture, s'engagent
} 
souci que sa sexualité lui a valu, et ce, depuis un très jeune âge. Dans un article que lui a consacré le New York Times en 2012, il évoque ainsi un événement survenu alors qu'il avait douze ans : un soir, des hommes ivres de son quartier se sont rassemblés devant sa maison, priant le jeune garçon de descendre pour leur accorder des faveurs sexuelles. Il se rappelle alors avoir eu l'impression que toute une foule était témoin de sa différence : «We all heard them. The whole family. The whole neighborhood. The whole world. ${ }^{2}$ Le sentiment d'exclusion qu'a dû ressentir l'auteur au moment des faits est d'ailleurs mis en évidence dès le titre qu'il donne à son témoignage, « $\mathrm{A}$ boy to be sacrificed $»^{3}$.

En partant de cet événement traumatique jusqu'au Printemps arabe, je propose ici d'interroger la manière dont Abdellah Taïa articule sa propre marginalité en rapport au collectif et particulièrement aux foules issues de mouvements populaires. À cet égard, le terme foule concernera donc dans cette étude tous les groupes de personnes dont le rassemblement a un sens et un impact politiques, qu'ils soient en faveur des régimes en place ou non.

Malgré sa première expérience d'exclusion et son immigration en Suisse puis en France en 1999, Taïa apparaît comme un acteur majeur dans la promotion du changement politique et social dans sa région d'origine. Au lieu de considérer sa singularité (en tant qu'homosexuel et immigrant) et son engagement politique au sein des mouvements

dans un « acte de contestation et de refus de demeurer dans l'ombre et dans le silence de leurs communautés » (2002, p. 105).

2 « Nous les avons tous entendus. La famille entière. Le quartier entier. Le monde entier» (je traduis)

3 « Un garçon à sacrifier » (je traduis) 
populaires comme étant surprenants, voire antithétiques, on montrera que la combinaison de ces deux facteurs offre la possibilité d'envisager les actions collectives comme garantissant le respect des particularités de chacun. Il convient pour cela de mobiliser le concept de multitude, que Michael Hardt et Antonio Negri définissent comme une foule composée de singularités qui agissent sur la base de ce qu'elles ont en commun $(2004$, p. 100).

En tant qu'outil conceptuel, la multitude invite à penser la relation entre l'homosexualité et les mouvements du Printemps arabe selon les axes suivants: Dans quelle mesure l'engagement de Taïa peut-il être perçu comme faisant partie des foules qui ont marché dans les rues de Tunisie, d'Égypte et d'autres pays de la région qui se sont révoltés contre leur régime? Peut-il être considéré comme agissant au sein ou en marge des foules? Comment l'homosexualité influence-t-elle sa vision des foules et comment les foules elles-mêmes peuvent potentiellement considérer une telle vision lorsque l'homosexualité est, dans leur pays, tabou dans l'espace public, voire illégal?

Suivant l'intuition d'Albert Memmi qui, dans Le Nomade immobile (2003), estime qu'être minoritaire offre une distance critique privilégiée, on montrera non seulement dans ses prises de positions politiques mais également dans son œuvre fictionnelle que Taïa jouit d'un avantage dans l'observation des foules et que son rôle vis-à-vis d'elles évolue. Alors que son roman Le Jour du Roi (2010) traduit le scepticisme de l'auteur quant à la possibilité d'un mouvement contestataire uni, Taïa montre dans Infidèles (2013), à la suite du Printemps Arabe, 
une volonté de passer d'une position d'observateur externe et sceptique à une position plus participative au sein des foules.

\section{L'homosexualité et le problème de sa réception dans le monde arabe}

Pour mieux comprendre la relation conflictuelle qu'entretient Abdellah Taïa avec son pays d'origine et la réception de son œuvre, il importe avant tout d'examiner la situation générale de l'homosexualité dans l'histoire du monde arabe. Max Kramer, à l'instar d'autres chercheurs s'intéressant aux sexualités marginales dans les sociétés musulmanes ${ }^{4}$, préconise d'abord d'approcher un tel sujet en tenant compte de la distinction entre pratique et identité sexuelles, étant donné que les pratiques homo-érotiques ne conduisent pas dans ces régions à un processus d'autodéfinition en fonction de cette sexualité (2008, p. 187). Cette discontinuité entre pratique et identité sexuelles expliquerait ainsi le constat fait par le journaliste Brian Whitaker qui, dans Unspeakable Love (2006), décrit à la fois l'aspect courant des rapports entre personnes de même sexe et l'absence de leur condamnation (p. 10). Puisque les actes se déroulent au sein d'un espace privé n'aboutissant pas à la reconnaissance publique d'une identité homosexuelle, nul besoin est alors de les condamner. Malek Chebel rappelle néanmoins qu'absence de condamnation ne signifie pas absence d'interdictions, notamment dans les lieux de

\footnotetext{
4 Parmi lesquels on citera Malek Chebel ou encore Joseph Massad.
} 
promiscuité tels que le hammam 5 (1988, p. 30). Cependant, ces interdits mis en place par la société sont parties prenantes d'un jeu extrêmement subtil «qui consiste à nier vigoureusement par les écrits et les exégèses les plus sophistiquées ce qui, tous les jours, se commet dans la stricte banalité des faits » (ibid., p. 30). Cet accommodement de la sexualité, y compris celle entre personnes de même sexe, diffère largement de celui des sociétés occidentales, qui ont tendance à privilégier une approche de l'homosexualité en fonction de l'orientation sexuelle et de l'identité, qui à terme permet l'émergence de discussions sur la culture gay et de la lutte pour la reconnaissance des droits civils (Kramer, 2008, p. 204). Le monde arabe, au contraire, bien que ne réprimant pas directement les actes, se montre réticent à l'émergence d'une parole publique homosexuelle au prétexte qu'elle serait une importation occidentale :

Homosexuality is a subject that Arabs, even reform-minded Arabs, are generally reluctant to discuss. If mentioned at all, it's treated as a subject for ribald laughter or (more often) as a foul, unnatural, repulsive, un-Islamic, Western perversion. Since almost everyone agrees on that, there is no debate. ${ }^{6}$ (Whitaker, 2006, p. 9)

5 Chebel évoque la notion d'Ih' yâ (le Pudique), qui met en place un double interdit dans les hammams. Le premier interdit, dit horizontal, réprime la communication entre les deux sexes, et le second, dit vertical, défend les comportements indécents entre les personnes de même sexe.

6 « L'homosexualité est un sujet que les Arabes, même les plus réformateurs, sont réticents à aborder. Si jamais l'homosexualité est mentionnée, elle est considérée comme un sujet pour plaisanteries grivoises ou (le plus souvent) comme une perversité occidentale odieuse, contre-nature, répulsive et non islamique. Puisque tout le monde tombe d'accord sur ce sujet, il n'y a pas de débat. » (je traduis) 
Malgré l'absence de débat ouvert dans le contexte actuel, les expressions et représentations de l'homosexualité ont pourtant eu leur place dans la littérature arabe. Samir Patrice El Maarouf rappelle que l'on peut remonter jusqu'au VIIIe siècle et au poète arabo-persan Abou Nawas pour trouver l'expression de désirs homosexuels. Nawas mais aussi d'autres auteurs comme Muhammad Al Nawadji, poète du XVe siècle, célébraient la virilité et le désir qu'elle suscite (El Maarouf, 2014, p. 165). Historiquement, la marginalité et l'ambiguïté sexuelles sont parties prenantes de la civilisation arabe. Le personnage de l'éphèbe, que l'on trouve, dans les textes coraniques, aux côtés du Prince (Chebel, 1988, p. 15) ou dans l'au-delà comme créature parfaite et sensuelle, amène à relativiser la notion de norme telle qu'elle peut être envisagée dans le monde musulman (Zekri, 2008, p. 167). Ainsi, la position actuelle du monde arabe décrivant l'homosexualité comme une perversion venant de l'Occident non seulement semble douteuse mais rend compte de la tension particulière qui, aujourd'hui, émerge autour de cette question, entre expression privée et publique. On peut, en effet, avancer que, pour le monde arabe, ce n'est pas l'homosexualité en tant que telle qui importe, mais plutôt la régulation de son expression dans l'espace public.

Avant le $\mathrm{XX}^{\mathrm{e}}$ siècle, la littérature arabe classique ne s'adressait pas à un public de masse. Pour la plupart, les textes érotiques étaient lus dans des cercles privés sans aucune intention de les publier par la suite (Whitaker, 2006, p. 88). Une telle intimité garantissait une plus grande liberté aux expressions à sensibilité homosexuelle. Par ailleurs, Zekri justifie la tolérance, dont Nawas a pu par exemple bénéficier, 
par le fait que l'Umma7 de son époque se trouvait en position de force (2008, p. 166). Or, la modification de ces deux facteurs explique sans doute pourquoi la représentation de l'homosexualité est devenue un problème dans le monde musulman et des auteurs tels qu'Abdellah Taia ont pu faire l'objet de vives critiques.

Quant à la littérature actuelle, du fait du développement des maisons d'édition et de la distribution, elle touche un public plus large, loin de se restreindre à une élite littéraire. Le passage d'un accès limité pour une élite proche du régime à une circulation à grande échelle parmi une masse plus difficilement contrôlable devient alors une menace pour les régimes en place, soucieux de préserver leur contrôle sur la société. Dans son article "Abdellah Taïa, homosexuel envers et contre tous" (2007), Karim Boukhari montre en quoi l'indignation que l'auteur suscite vient avant tout de son importante médiatisation et essentiellement de son coming out paru en février 2006 dans le magazine TelQuel ${ }^{8}$. La publication par la suite des entretiens dans lesquels Taïa parle de son homosexualité dans les titres arabophones intensifiera de manière significative les critiques, car l'arabe, considéré comme la langue de tous, étend la réception et donc l'impact de sa révélation. Pour Boukhari, c'est à partir de ce moment-là que «le jeune homme est devenu "l'autre", l'extra-terrestre, le clochard montré du doigt par les gosses de Hay Salam, le héros monstrueux des contes transmis par les grand-mères à leurs petits-enfants ». On voit alors que la marginalisation d'Abdellah

\footnotetext{
7 Terme désignant en arabe la communauté.

${ }^{8}$ Son coming out sera aussi relayé dans l'ensemble de la presse francophone marocaine tels que Le Journal, Maroc-Soir ou encore Maroc Hebdo.
} 
Taia se développe à mesure que son lectorat s'élargit. Boukhari écrit d'ailleurs que s'il est encore épargné par la censure, c'est que, selon un «dirigeant du parti islamiste [...]: "Taïa ne représente pas grand-chose, il ne vend pas - encore - assez de livres" » (ibid.).

Outre le lien entre médiatisation et marginalisation, de tels propos montrent le rôle politique que peut jouer Taïa en tant qu'écrivain ouvertement homosexuel. Il représenterait à terme, aux yeux du pouvoir en place, un danger pour l'unité de l'Umma. Dans Desiring Arabs (2007), Joseph Massad explique la possibilité d'un tel ressenti dans les sociétés musulmanes du fait de l'interventionnisme occidental sur les concepts de désir et pratique sexuels depuis la fin des années 1960 (2007, p. 160). L'une des formes de cet interventionnisme, qu'il identifie et nomme l'«Internationale Gay», consiste à promouvoir une identité gay universelle qui prend ses fondements épistémologiques en Occident (ibid., p. 162) et qui se transmet par le tourisme sexuel, les programmes télévisés, Internet et les organismes LGBT (Kramer, 2008, p. 162). La méfiance que les sociétés musulmanes ressentent vis-à-vis de l'homosexualité s'expliquent directement par ces activités. Elles seraient responsables non seulement du rejet de certaines formes de désirs entre personnes de même sexe en les réorientant selon des termes proprement occidentaux mais également de l'émergence en retour d'une rhétorique et de pratiques qui s'inscrivent en contrepoint de ce qu'elle visait (Massad, 2007, p. 180). Il est, en effet, intéressant d'observer qu'en parallèle avec le silence imposé sur tout débat potentiel concernant les identités sexuelles dans le monde arabe, se soient multipliés les discours insistant sur le danger que représentent ces identités dans l'espace public. 
Si la responsabilité du rejet de la marginalité sexuelle incombe en grande partie aux lobbys occidentaux, elle touche également ceux que Massad appelle les «native informants », que l'on peut traduire par «informateurs indigènes » (ibid., p. 172). Venant, toujours d'après Massad, des classes moyennes et supérieures, ces informateurs soutiennent, au travers de leur engagement dans des organisations internationales, l'interventionnisme occidental sur leur pays d'origine au nom de la liberté sexuelle. Au vu des positions tenues par Abdellah Taïa sur son homosexualité et de son immigration en Suisse puis en France, l'amalgame entre son rôle d'écrivain et celui de l'«informateur indigène » établi par Massad est tout à fait envisageable. Même s'il est issu d'un milieu populaire et ne s'est jamais engagé dans une organisation internationale promouvant les droits LGBT, Taïa est perçu comme un allié $\mathrm{du}$ monde occidental pouvant potentiellement, du fait de son homosexualité, menacer les valeurs et l'ordre public de son pays d'origine. Boukhari retranscrit entre autres dans son article certaines critiques qu'il a pu entendre dans des conversations de rue ou en ligne à l'encontre du jeune auteur : «Il se prostitue pour plaire à l'Occident», «C'est son postérieur qui parle, pas lui », "Il nuit à l'image du Maroc et de l'islam » (Boukhari, 2007).

\section{De l'utilité de l'homosexuel comme bouc émissaire ou comment brandir la menace sociale pour mieux diviser et étouffer les éventuels collectifs résistants}

Bien que le lien de cause à effet établi par Massad entre interventionnisme occidental et rejet des sexualités marginales par les sociétés musulmanes soit crucial, il est essentiel de voir 
qu'un tel rejet peut tourner aussi à l'avantage des régimes en place dans le monde arabe. En assimilant les homosexuels à des agents de l'étranger, ces derniers parviennent ainsi à limiter, voire à faire taire toute possibilité contestataire émanant des marges au sein même de leur société, sous prétexte de menace d'ingérence étrangère. Chebel rappelle à ce propos que lorsqu'elle est reconnue par les sociétés musulmanes, la marginalité «est du même coup suspectée, chassée avec intransigeance et parfois rendue responsable de nombreuses incursions dans les mœurs de la majorité conventionnelle ${ }^{9}$ » (1988, p. 17). Il convient donc de relativiser les accusations de collusion avec l'Occident portées à l'encontre d'individus en marge et considérés comme opposants au régime, étant donné tous les avantages qu'une telle stigmatisation offre aux pouvoirs en place, soucieux de conserver l'ordre qu'ils ont euxmêmes défini.

Le raid de la police mené en 2001 sur le "Queen Boat», une discothèque du Caire dans laquelle les homosexuels avaient pour habitude de se retrouver, illustre particulièrement bien cette stratégie de stigmatisation et de division en faveur du maintien du pouvoir en place et du statu quo social. À la suite de la descente de police, une cinquantaine d'hommes furent arrêtés et jugés publiquement devant une cour de sécurité de l'État spécialement mise en place pour traiter des cas de terrorisme, au lieu de paraître devant une cour ordinaire. Bien que Massad justifie ce choix, d'une part, par l'absence en droit

\footnotetext{
${ }^{9}$ Si Chebel (1988, p. 19) reconnaît cette réinsertion de la marginalité en tant que bouc émissaire dans le contexte contemporain, il rappelle toutefois qu'un tel usage avait déjà cours dans le passé. Il évoque notamment l'exemple du penseur Ibn Khaldoûn, qui avait fait de l'homosexualité l'un des facteurs de la corruption des mœurs sociales de son époque.
} 
égyptien de disposition à l'encontre des pratiques sexuelles entre personnes de même sexe et, d'autre part, par le fait que l'Égypte est placée en état d'urgence depuis les années 1980 (2007, p. 182), il est difficile de nier qu'une telle décision facilite l'assimilation entre homosexuels et terroristes. Cette désignation de l'homosexualité comme l'une des menaces les plus dangereuses pour la nation a également trouvé écho dans la presse, par exemple dans un journal du Caire titrant,

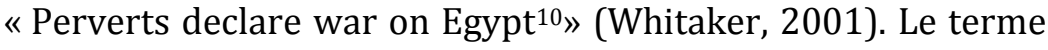
« déclaration de guerre » ajouté à l'idée que l'homosexualité est une perversion occidentale suggère alors que les homosexuels sont perçus comme une force étrangère qui peut s'attaquer à la culture égyptienne ${ }^{11}$.

Dans un article du Guardian datant du 19 novembre 2001, Whitaker remarque par ailleurs l'usage dans la presse de la métaphore de l'infection. L'homosexualité est décrite comme une maladie que les Égyptiens peuvent contracter s'ils ne font pas attention. Bien que cette peur de la contagion puisse relever en partie d'une crainte irrationnelle proprement homophobe, elle suggère également, il me semble, la peur d'une force collective qui pourrait remettre en cause les fondements jusqu'alors établis par le régime en place. Dans Crowds and Politics (2014), ouvrage consacré aux foules du Printemps arabe, Andrea Khalil rappelle l'importance en Tunisie d'un soi collectif se développant justement par contagion et rejetant les

\footnotetext{
10 « Les pervers déclarent la guerre à l'Égypte. » (je traduis)

11 Pour une étude détaillée de l'affaire du Queen Boat, et notamment l'escalade de l'opposition entre des organisations internationales qui opèrent une couverture médiatique sans précédent et la presse égyptienne qui dénonce l'impérialisme cherchant à imposer une déviance dans leur société, voir Massad (p. 184).
} 
divisions de la dictature (p. 27). En refusant l'atomisation de l'individu moderne du fait des dichotomies établies par l'État, la foule devient le terreau idéal d'une force collective unie dans la contestation puisque ses limites et ses possibilités d'expansion sont infinies. Khalil explique cette force expansive en proposant le concept de "la personne comme foule», largement inspiré des approches poststructuralistes de Gilles Deleuze et Félix Guattari. Selon la configuration de "la personne comme foule », «the individual is not the opposite of crowds, or a structural model for a crowd, but rather already exists as a crowd, in a constitutive coexistence within a crowded field ${ }^{12}$ » (Khalil, p. 26). Étant donné que les individus sont, d'après ce modèle, déjà ensemble dans la foule, le fait qu'ils se rassemblent en collectif les place dans un «devenir humain » ou un «devenir soi-même » qui demeuraient jusque-là à l'état embryonnaire. Puisqu'un tel concept s'adresse à n'importe quel individu, il représente une menace significative pour les régimes autoritaires qui étendent leur pouvoir grâce aux divisions qu'ils créent. Dans le cas de l'homosexualité, il est possible de comprendre ainsi que la stigmatisation des homosexuels participe à la stratégie de division des individus et, particulièrement, de ceux qui sont mis à la marge et qui ont, en conséquence, plus de chance de se révolter contre le régime en place.

C'est ce qu'Abdellah Taïa, lui-même victime de ce type de stigmatisation, cherche à remettre en cause dans son texte "L'homosexualité expliquée à ma mère », publié en avril 2009 dans le magazine marocain TelQuel. S'adressant à sa famille,

12 «l'individu n'est ni l'opposé des foules, ni un modèle structural pour une foule, mais plutôt existe déjà lui-même en tant que foule, dans une coexistence constitutive dans un espace lui-même bondé » (je traduis) 
l'auteur y décrit son refus de la marginalisation et sa volonté de communiquer malgré sa différence : "Je rêve du dialogue. Un dialogue impossible jusqu'à aujourd'hui. Je ne suis pas dans la minorité. Je suis vous, avec vous, toujours avec vous, même quand je brise les tabous » (je souligne). Bien qu'elles soient adressées à sa famille, ces lignes, qui sont publiées, dépassent alors le cadre strictement personnel et concernent, si ce n'est le monde arabe dans son entier, du moins le Maroc. Les homosexuels font, selon lui, partie d'une révolution qui est vouée à éclater et qui ne peut que se faire dans «la provocation et le scandale». Cette singularité n'entre pas, pour Taïa, en contradiction avec sa volonté de s'inscrire dans un mouvement collectif. Sa position s'avère, en effet, très similaire à celle avancée par Michael Hardt et Antonio Negri, qui voient dans la multitude la possibilité pour chaque singularité d'agir sur la base de ce qu'elles ont en commun (2004, p. 100). Les homosexuels représentent ainsi pour Taïa un des groupes qui agissent pour le changement et qui sont la multitude, avec la multitude, toujours avec la multitude. Par ailleurs, Taïa ne se bat pas forcément pour la reconnaissance des droits homosexuels mais promeut plutôt le changement dans la société marocaine, qui concerne et inclut tout le monde :

Je ne supporte plus qu'on ne voie pas la richesse réelle de ce pays : l'imaginaire, les histoires, le mystère. LA JEUNESSE. Je ne supporte plus qu'on n'aide pas assez le Maroc à se relever et à grandir. Je ne supporte plus ce système qui casse du matin au soir le Marocain et qui fait taire les voix nouvelles qui émergent pour dire ce pays autrement. Je ne supporte plus cette médiocrité et cette petitesse qu'on nous impose. Le Maroc est, pour moi, plus grand que tout cela. (Taïa, 2009)

L'éloignement géographique de l'auteur à la suite de son immigration, pas plus que la marginalisation sexuelle, ne 
constituerait une limite à son inclusion dans la multitude, selon Hardt et Negri. Ces derniers défendent au contraire l'idée selon laquelle les migrants doivent être pensés en fonction de valeur collective : "Migrants demonstrate (and help construct) the general commonality of the multitude by crossing and thus partially undermining every geographical barrier.13» (2004, p. 134) En combinant les influences qu'il reçoit de sa vie en France à celles de ses origines, Taïa remet aussi bien en cause la stratégie des régimes en place, qui établissent des frontières strictes entre l'Occident et le monde arabem que le modèle de l'informateur indigène imposant les valeurs occidentales à son pays d'origine. Pour Gibson Incube, l'exil, chez Rachid O. comme chez Abdellah Taïa, enclenche « un phénomène de méditation et de médiation de leur "sexualité" par un perpétuel "retour au pays natal” » (2014, p. 128). On comprend ainsi qu'au lieu d'accentuer sa différence vis-à-vis de son pays d'origine, Taïa cherche à instaurer au contraire un dialogue jusque-là impossible et à montrer que sa singularité participe à la construction d'un collectif.

\section{Tension entre un désir de collectif et un besoin d'émancipation individuelle}

Même si, dans "L'homosexualité expliquée à ma mère", il existe chez Taïa un fort désir de s'engager dans un collectif à partir de la reconnaissance de sa propre singularité, ce désir ne se concrétise pas dans un projet qui articulerait de manière précise comment l'auteur peut participer et agir dans le

13 «Les migrants montrent (et aident à construire) les similitudes de la multitude en traversant et ainsi en remettant en cause partiellement chaque barrière géographique » (je traduis) 
collectif. Partager un rêve commun et un projet avec un collectif potentiel ne revient pas au même que d'être un agent de ce collectif. De plus, même s'il est possible, comme nous l'avons fait, de relativiser la marginalité de Taïa en raison de son homosexualité et de son éloignement géographique, il s'avère tout de même difficile d'imaginer de manière concrète son inclusion dans une foule qui vit encore au Maroc et a intériorisé l'homosexualité comme une identité occidentale et perverse. Par ailleurs, malgré les élans collectifs de «L'homosexualité expliquée à ma mère », Taïa reconnaissait lui-même auparavant que «ce n'est que dans l'individualité que l'on peut faire avancer les choses et être soi-même» (Boukhari 2007). Le critique Khalid Zekri estime, lui aussi, à propos d'auteurs comme Taïa ou Rachid 0., que leurs textes qui remettent en cause les définitions légitimes et dominantes de l'hétéronormativité ne s'inscrivent plus dans un destin collectif mais plutôt dans une "expérience individuelle qui tente de se dire malgré la coercition qu'exerce le discours dominant sur le sujet de l'énonciation» (2008, p. 174). Faut-il donc voir, dans le passage de l'interview de 2007 au texte de 2009, seulement un changement de rhétorique de la part de Taïa? Ou peut-on voir les premiers jalons d'une démarche alliant l'individu au collectif?

\section{Du rôle d'observateur minoritaire...}

Pour aborder ces questions, Samir Patrice El Maarouf offre une perspective intéressante en s'appuyant sur Le Nomade immobile (2003) d'Albert Memmi, dont il cite un passage en épigraphe : 
Je suis convaincu que l'on voit mieux de l'extérieur, comme on aperçoit mieux une foule du haut d'une terrasse. C'est un aspect positif de la condition minoritaire [...] : elle oblige à considérer, à distance, les événements, les gens et leurs alibis. (2014, p. 177)

El Maarouf tombe en effet d'accord avec Memmi pour dire que faire partie d'une minorité offre un avantage significatif pour observer les mouvements collectifs et précisément les foules. Le rôle de l'observateur suppose une distance critique qui lui permet de réfléchir sur les dynamiques de la foule et de ses rapports aux événements et au pouvoir. En tant que minorité, Taïa endosse parfaitement ce rôle et participe au développement de ce qu'El Maarouf appelle une « éthique de la marge » (p. 177).

Dans Le Jour du Roi (2010), Taïa, en tant qu'observateur / auteur, propose une analyse très détaillée de la foule à partir du point de vue de son protagoniste, Omar. Tout au long du roman, ce dernier fait l'expérience de sa singularité par rapport aux autres. Dans le premier chapitre, il rêve par exemple que le roi du Maroc lui demande son nom de famille et la date à laquelle il a accédé au trône, deux questions auxquelles tout citoyen dévoué est censé répondre sans hésitation. Or, Omar, lui, hésite beaucoup et en vient même à confondre la date de l'indépendance (1956) avec celle de l'accès au pouvoir d'Hassan II (1961). Alors que le roi lui pose ces questions, Omar est entouré par une foule qui se moque de ses hésitations et qui se conforme aux différents changements d'humeur manifestés par le monarque : "Il [le Roi] se lève, claque des doigts, trois fois. Tout le monde baisse la tête et se cache les yeux avec les mains. Sauf moi. J'imite les autres, je baisse la tête sans fermer les yeux. » (Taïa, 2010, p. 15), « Le Roi rit plus fort. La salle, 
toute la salle l'imite alors.» (ibid., p. 21) Dans la première citation, le comportement singulier d'Omar apparaît d'abord dans le fait qu'il ne maîtrise pas le code de conduite que tout le monde connaît parfaitement et, ensuite, quand, contrairement aux autres, il ne ferme pas ses yeux. Cette seconde particularité suggère le potentiel contestataire présent chez Omar. Sa soumission n'est pas totale. Dans la seconde citation, le rire qui se répand du roi à la foule est de type bergsonien puisqu'il sert à condamner socialement l'ignorance qu'Omar a du régime dans lequel il vit: «Bye-bye... Tu n'es plus marocain... Tu n'es plus marocain... Bye-bye... Tu n'as plus de père... Bye-bye... Tu n'as plus de père... Bye-bye... Tu n'as plus de Roi... » (ibid., p. 22) À la fin du rêve, Omar est déchu de sa nationalité et privé de tout point de référence. Ce chapitre introductif donne la tonalité qui sera celle du roman dans son entier en préparant la montée en puissance du potentiel contestataire envers le Roi et la foule.

Le rêve d'Omar s'avère d'autant plus subversif qu'il est en lien avec le réel vécu par le protagoniste et ses concitoyens : le roi est, en effet attendu dans leur ville, Salé. Le roman raconte l'histoire de cette attente pour la célébration du roi, attente qui donne à Omar et à son meilleur ami Khalid le temps de réfléchir sur le sens d'une telle célébration et sur son possible échec. Le caractère dissident du rêve est par ailleurs confirmé plus loin dans le roman, lorsqu'Omar le raconte à Khalid :

- J'avais l'impression qu'il était... qu'il était...

- Quoi ? Quoi?

- Qu'il n'était plus le Roi. Mais cela a duré à peine une seconde ou deux. Une minute ou deux... C'était au milieu du rêve. Mais cette partie n'est pas la plus importante. Il s'est passé quelque chose d'autre, de plus terrifiant. Un vrai cauchemar.

$[\ldots]$ 
- Oui, oui je me souviens de tout. Ce n'était pas un rêve. C'était réel. (ibid., p. 77)

Le fait qu'Omar se confie à Khalid prend une importance toute particulière puisque ce dernier est celui qui a été désigné pour accueillir le roi à Salé. La nomination de Khalid par le directeur de l'école est vue par Omar comme une trahison qui le sépare de son ami : "Khalid s'éloignait. Volait loin. Loin de nous. De moi. Khalid vivait le rêve. Son rêve? Le rêve de tout Marocain? » (ibid., p. 90) Le «nous », ici, inclut la mère d'Omar, qui, en décidant d'abandonner son fils et de revenir à ses origines, dans son village, représente la résistance, le refus du rêve que tout Marocain est censé avoir. Ainsi, à mesure qu'il se rapproche de sa mère, Omar accentue sa différence avec les autres Marocains, y compris Khalid. Il se voit lui aussi différent du reste de la foule. Cependant, les interrogations à la fin de ce passage signalent son hésitation vis-à-vis de la foule et de son ami. Il semble se demander si la rencontre avec le roi est aussi réellement le rêve de son ami et du peuple marocain.

Si la séparation entre lui et le reste de la foule peut paraître moins significative qu'elle n'y paraît, Omar réalise cependant que, contrairement au peuple qui attend patiemment le roi, il ne se soucie guère d'Hassan II. Il considère les chansons patriotiques, entonnées par la foule, dénuées de sens et de saveur. La répétition des mêmes rituels l'exaspère (ibid., p. 107). Au moment de la célébration, Khalid s'exclut lui aussi de la foule. L'amitié qui le lie à Omar explique sans doute pourquoi il finit par ne pas se conformer à ce qui était attendu de lui. Omar confirme ce sentiment d'exclusion dont tous deux font l'expérience quand il affirme: «Nous devions garder le silence. Montrer notre respect. Croire tous les deux en les 
mêmes valeurs. Rejoindre le peuple marocain uni et fier qui attend son roi. Nous avons fait l'inverse. Malgré nous, nous avons suivi un autre Dieu, un autre maître, un autre diable » (ibid., p. 108), ou encore «la foule ce n'était pas nous » (ibid., p. 111).

La différence de Khalid et de Omar avec le reste du peuple est, par ailleurs, largement influencée par leur relation homoérotique. Tout au long du roman, Taïa insiste sur l'aspect fusionnel de leur relation : «Il avait l'habitude. De moi. De mon corps. De nous. Deux. Un. » (ibid, p. 44) Dans le dernier chapitre, Hadda, la servante de la famille de Khalid, décrit son admiration pour l'amour qu'entretiennent les deux garçons et qui les mènera vers une nouvelle histoire, dans laquelle elle s'inclut aussi :

J'entrais dans la chambre de Khalid et je les regardais dormir collés l'un à l'autre, l'un dans l'autre. Leur amitié semblait forte, indestructible, en dehors des règles, et c'est ce qui me plaisait. Ils étaient à part. Ils écrivaient une histoire à part. Je rêvais avec eux. Pour eux. Loin d'eux. De plus en plus. (ibid., p. 203; je souligne)

Le témoignage d'Hadda montre que l'amitié particulière des protagonistes, hors des règles, est une source d'inspiration qui ouvre un nouveau chemin qu'elle peut suivre également, même loin d'eux. La contagion d'une liberté née d'une relation homosexuelle ne requiert pas le parallélisme total d'une telle relation. Inspirée par Omar et Khalid et voulant les suivre dans cette fondation d'une nouvelle histoire, Hadda ne les imite pas pour autant. On notera toutefois dans cette citation la répétition de l'expression «à part », qui oppose à nouveau les deux héros au reste du peuple et semble alors limiter le potentiel de contagion aperçu par Hadda. Il est possible de comprendre 
cette sensation d'être «à part » comme celle-là même ressentie et vécue par Taïa. Les éventuelles actions révolutionnaires reposent sur les individus qui s'excluent eux-mêmes de la masse. D’après ce modèle, Taïa semble alors confirmer la division établie par le régime en place, qui considère les homosexuels comme étant une menace pour le reste de la population qui vit paisiblement sous la monarchie marocaine.

\section{... à celui d'acteur à part entière au sein de la multitude}

Écrit en 2010, par conséquent avant le Printemps Arabe, Le Jour $d u$ Roi montre le scepticisme de Taïa envers la capacité d'une multitude à renverser le régime en place et à créer une nouvelle voie pour le monde arabe. Il privilégie, en effet, la voix d'un individu à la voix d'un collectif. Dans la préface d'Égypte, les martyrs de la révolution (2014), Taïa reconnaît qu'avant le Printemps Arabe, il ne pensait pas qu'un mouvement contestataire collectif était possible: «Je ne voyais pas d'où pouvait venir le vent fort d'une émancipation, à la fois collective et individuelle» (p.11). Il explique entre autres son mauvais jugement par le fait qu'il avait été aveuglé par les idées des autres, que ce soient celles des régimes du monde arabe ou de l'Occident. En admettant sa propre assimilation des divisions, Taïa franchit la première marche qui mène vers la construction de la multitude. Il préfère désormais voir, au-delà des différences, les similitudes, les points communs. C'est ainsi qu'on observe dans sa position comme dans son écriture le passage du rôle de simple observateur au rôle d'agent au sein de la foule. Dans un hommage adressé à Mohamed Bouazizi, l'homme qui s'immola en signe de contestation et qui est à 
l'origine de la Révolution en Tunisie, Taïa expose de manière spécifique le rôle qu'il a l'intention de jouer dans cette révolution, celui d'écrivain :

Je suis écrivain, cher Mohamed. Je sais que mon devoir est d'être avec ces révolutionnaires. D'être fort avec eux. Alors j'écris. À toi. Et ailleurs. Écrire pour marquer ce mouvement. Le voir et le revoir. Le porter haut. Le porter grand. Je me libère grâce à toi, grâce à eux, à ces frères et sœurs dans la révolte. Nous n'écrirons plus comme avant dans le monde arabe. Nous ne penserons plus comme avant. Tout change. Tout bouge. (Taïa, 2011)

Il est intéressant ici de remarquer le passage du « je » au «nous » en lien avec la libération personnelle. Taïa révèle que, loin de seulement observer les actions des autres, il a trouvé des frères et des sœurs qui l'ont inspiré à rejoindre la lutte. En s'intéressant maintenant au premier roman qu'il a publié après les événements du Printemps arabe, il importe de voir dans quelle mesure une telle révélation a influencé son écriture.

$\mathrm{Au}$ premier regard, Infidèles semble jouer sur le même registre que Le Jour $d u$ Roi, mettant l'accent sur l'opposition entre des singularités marginales et le reste du peuple. Le jeune protagoniste, Jallal, ressent ainsi le besoin de protéger sa mère, une prostituée, des autres habitants de son village qui la rejettent : "Je crache sur cette ville, Salé, et sur tous ceux qui ne te [la mère] reconnaissent pas. ${ }^{14}$ » (Taïa, 2012, p. 13) La mère de Jallal représente, pour Taïa et son protagoniste, un personnage qui est une figure centrale dans la société. Elle est

\footnotetext{
${ }^{14}$ " Je crache » est par ailleurs répété à plusieurs reprises tout au long de ce premier chapitre, augmentant un peu plus l'effet d'opposition entre le protagoniste et la foule.
} 
celle qui garantit le respect des traditions tout en connaissant l'hypocrisie qui les soutient. En effet, Slima est chargée d'enseigner le sexe aux hommes le jour de leur mariage et de s'assurer que le sang se remarquera sur les draps blancs. Prenant part au moment le plus intime et sachant ce que tout le monde ignore, Slima est « un être à part. Plus qu'aujourd'hui. Plus que les autres » (ibid., p. 47). Par la suite, Slima est torturée dans le sud-Maroc, car elle refuse de donner des informations sur un soldat dissident qui avait refusé de combattre contre le Polisario ${ }^{15}$ et qui était, en conséquence, considéré comme un traître. Une fois libérée, elle se place plus que jamais en résistante vis-à-vis du pouvoir en place en affirmant qu'il n'y a plus de vrais musulmans au Maroc mais seulement d'obéissants esclaves assoiffés de sang (ibid., p.111). Ce constat, quoique plus sévère et violent, rappelle celui tiré par Omar dans son rêve dans Le Jour $d u$ Roi, lorsqu'il se plaint d'avoir devant lui une foule aveugle et obéissante. La possibilité d'être déchu de sa nationalité est un autre point commun avec le rêve d'Omar : «Brûler mon passeport» dit Slima, «Brûler ma carte d'identité marocaine. Renaître pour toi, Jallal. Pour nous. M'accrocher à ce rêve : Marylin » (ibid., p. 111).

Pris en dehors de son contexte, le rêve que représente Marylin Monroe pourrait sembler montrer un certain désir pour l'Amérique, une échappatoire possible du Maroc. Même s'il est vrai que Slima aspire à se rendre dans un autre pays garantissant plus de liberté (elle envoie d'ailleurs Jallal en Égypte), on remarquera que la référence à Marylin Monroe sert

\footnotetext{
15 Le POLISARIO, provenant de l'abréviation espagnole Frente Popular de Liberación de Saguía el Hamra y Río de Oro (Front Populaire de Libération de Saguía el Hamra et Río de Oro), est un mouvement national qui a combattu pour la libération de l'Ouest-Sahara, occupé par le Maroc.
} 
moins à insister sur les différences entre l'Occident et le monde arabe qu'à en montrer les points communs. La première similitude est établie par Jallal, qui établit un parallèle entre Marylin et sa mère. Elles sont toutes deux charismatiques, adulées et pourtant seules, parmi une foule, et pourtant singulières : "Les âmes se rencontrent, se reconnaissent et se parlent même quand les mers, les océans les séparent. » (ibid., p. 69) Marylin et Slima sont des singularités qui peuvent parler aux autres, à la multitude. Même le langage ne constitue plus une barrière : «Elle [Marylin] parle anglais et, dans les oreilles, mon cœur, c'est comme si c'était de l'arabe » (ibid., p. 69). Il est aussi important de signaler que Marylin est largement associée au film Rivière sans retour, référence culturelle centrale dans le roman. Jallal, qui a découvert le film grâce au soldat dissident, ne cesse de rappeler son obsession pour la manière dont est dépeinte la foule de cowboys et de marginaux à la recherche d'or :

C'est une foule sauvage, en rupture, à la recherche d'un moment fugitif de tendresse. On boit. Et on boit. Et on boit. Au milieu de ces hommes, un petit garçon, un petit homme. 10 ans. 11 ans peut-être. Il est chez lui ici, dans cette foule dangereuse, à la limite du désespoir. [...] Il a tout vu ici dans ce camp. Les assoiffés. Les désaxés. Les fous. Les saints. Les prostituées. Les prêtres. Les chanteuses. Les guerriers. Les morts. Les survivants. Les chefs. Marylin Monroe. (ibid., p. 72)

La liste hétérogène des personnes parties prenantes de cette même foule, conduite par un même idéal, représente pour Jallal un moyen d'imaginer une action collective en dépit des singularités de chacun. Il est aussi possible de voir dans l'identification du protagoniste au jeune garçon du film le développement de son rapport au collectif, puisque le garçon est décrit comme se sentant chez lui parmi cette foule. 
Devenant une source d'inspiration, le film prépare le terrain pour la dernière action du roman, mettant en scène Jallal et un jeune homme belge récemment converti à l'islam, Mahmoud. Séduit par ce dernier, Jallal accepte de préparer un attentat au Maroc. Le but de cette attaque, décrit par Jallal, semble illustrer la tension que Taïa ressent lui-même entre son opposition à une foule obéissante et son désir pour une multitude :

Donner à méditer un geste. Être contre cette plaie qui se répandait partout au Maroc. La banalité. L'étroitesse. L'enlisement. La soumission. L'enlisement dans le faux et l'ignorance. La destruction programmée des individus, de ceux et celles qui, comme ma mère Slima, osent un jour tenter la liberté, la résistance, une autre voie. S'élever contre tout un pays. Contre tout un peuple. Poser enfin les vraies questions. Qui nous a amenés jusque-là, à cette déchéance, à ce malheur, à cette négation de nous-mêmes, à cet aveuglement contagieux? Qui empêche nos âmes de voler et d'écrire une autre histoire avec un nouveau messager? Qui nous bloque, nous pétrifie et nous dénie le droit d'être ce que nous sommes à l'origine : des hommes debout? (ibid., p. 158)

Tout d'abord, l'attentat est, pour Jallal, un moyen de porter un message de résistance adressé au peuple. Or un tel désir d'impact sur la population diffère déjà de l'approche prise par Taïa dans son roman précédent, dans lequel les protagonistes s'excluaient eux-mêmes. À première vue, l'on pourrait certes avancer que l'acte de résistance pris dans le sens de lutte contre un peuple et un pays n'invite pas forcément à une lecture de cet acte comme voué au bien-être de la population générale. Cependant, une analyse attentive des idées développées dans le passage nous permet de considérer «la lutte contre» comme s'inscrivant davantage dans la voie résistante ouverte par Slima que dans la direction initialement prévue par Jallal et par Mahmoud. En effet, le «nous» qui 
émerge dans les questions suivant les affirmations de résistance n'inclut pas seulement les deux amis préparant l'attentat mais le peuple en général. Le « nous » s'adresse à ceux qui, à l'origine, étaient « des hommes debout». C'est le « nous » composé d'individus singuliers se rassemblant pour un idéal, le « nous » de la multitude. Le fait qu'à la fin du roman, l'attaque terroriste échoue renforce l'idée que Taïa ne voulait pas que ces personnages soient perçus comme des ennemis. Au lieu de tuer des gens, Jallal et Mahmoud se font exploser dans un cinéma désert, donnant à leur geste un impact symbolique plus significatif. Le choix d'un tel lieu rappelle également au lecteur l'importance du film Rivière sans retour, qui est l'inspiration première de Jallal dans sa révolte contre l'ordre et son expérience de la foule menée par un idéal.

Bien que soit attestée l'évolution des conceptions de Taïa sur l'individualité en relation au collectif, à savoir le passage du rôle d'observateur à celui d'agent partie prenante d'un collectif, un examen des derniers mots d'Infidèles offre un aperçu intéressant des limites que l'auteur semble encore connaître au regard de la multitude. Le ton prophétique de Jallal lorsqu'il prononce «d'un même mouvement, deux jumeaux en un seul corps, deux étrangers dans la même fois, Dieu unique, nous avons fait la lumière » (Taïa, 2012, p. 173), est, par exemple, indéniable. La relation de Mahmoud et de Jallal, extrêmement homo-érotique, à l'instar de celle décrite dans Le Jour $d u$ Roi, est présentée comme une fusion, un révélateur qui pourrait gagner l'ensemble d'une foule entière mais qui reste toutefois spécifique. Au lieu de considérer la formation d'une force collective dans laquelle chaque individu prendrait part de manière égale, Taïa privilégie l'idée d'un prophète éclairant la 
foule. Une telle configuration n'est pas fortuite mais décrit plutôt la manière dont il comprend son rôle en tant qu'écrivain en relation avec son pays et le monde arabe en général. Il se voit en même temps marginalisé et, par conséquent, dominé du fait de son homosexualité mais également capable, du fait de cette position même, d'ouvrir ses yeux sur l'oppression dont lui et d'autres souffrent, et ainsi d'inviter à la révolte. Dans « Espace social et genèse des "classes" » (1984), Pierre Bourdieu appelle cette position ambivalente, typique chez l'intellectuel, «agent dominé parmi les dominants» (p. 10). Un tel agent est dominé dans le champ du pouvoir et, par conséquent, conscient des effets de domination. Mais il est aussi dominant, comparé au reste de la population, étant donné qu'il est un producteur culturel.

En tant qu'auteur, Taïa est par conséquent capable d'offrir aux dominés les moyens de commencer une révolte et de penser un nouveau chemin pour le monde arabe. Mais comme Bourdieu l'explique, l'homologie de positions entre deux groupes différents est souvent la base d'alliances ambiguës puisque la reproduction de dynamiques de domination s'instaure. Le concept de multitude d'Hardt et de Negri remet en cause ces dynamiques de domination puisque la multitude est «rather than a political body with one that commands and others that obey», "living flesh that rules itself $^{16} »(2004$, p. 100). L'inclination de Taïa envers la multitude n'est donc dans un sens jamais totale, de la même manière que le Printemps arabe n'a pas drastiquement transformé la société arabe. Malgré ces limites, on retiendra

16 «plutôt qu'un corps politique avec quelqu'un qui commande et les autres qui obéissent », « une chair vivante qui se dirige elle-même » (je traduis) 
que le romantisme politique de Taïa reste prégnant : «I still believe in it, in this Revolution that came as such a shock, to so many people and to myself, when it occurred. I still refuse to fall into political realism that considers this political moment (in all senses of the term) to be dead to Arab lives. ${ }^{17}$ » (2014, p. 62) C'est avec ce genre de discours que Taïa invite ses lecteurs à rester optimiste et, plus important encore, à croire en l'idée d'une multitude jamais morte mais toujours en devenir et ayant pour objectif premier un futur meilleur.

\section{Bibliographie}

BOUKHARI, Karim. (2007), « Abdellah Taïa, homosexuel envers et contre tous », TelQuel, no 277, 9-15 juin, <http://fr.ossin.org/droits-de-l'homme/104-homosexualiteen-afrique/328-abdellah-taia-homosexuel-enver-en-contretous>.

BouRdiEU, Pierre. (1985), «Espace social et genèse des "classes" ", Actes de la recherche en sciences sociales, vol. 5253 , no 1 , p. 3-14.

Chebel, Malek. (1988), L'Esprit de sérail. Perversion et marginalités sexuelles au Maghreb, Paris, Lieu Commun.

DAILLEuX, Denis. (2014), Égypte, les martyrs de la révolution Textes d'Abdellah Taïa et de Mahmoud Farag, Marseille, Le bec en l'air éditions.

\footnotetext{
17 « J'y crois encore, en cette Révolution qui est venue comme un choc, pour de nombreuses personnes et moi-même, quand elle a eu lieu. Je refuse encore de tomber dans le réalisme politique qui considère que ce moment politique (dans tous les sens du terme) est mort pour les vies arabes. » (je traduis)
} 
El MaArouf, Samir Patrice. (2014), Les Prémices littéraires des révolutions arabes. Yasmina Khadra, Assia Djebar, Abdellah Taïa, Paris, L'Harmattan.

HARDT Michael et Antonio NEgRI. (2004), Multitude, War and Democracy in the Age of Empire, New York, The Penguin Press.

KhaliL, Andrea. (2014), Crowds and Politics in North Africa, Londres, Routledge.

KRAMER, Max. (2008), «L'orientation sexuelle: La percée de l'identité gay dans le monde musulman», dans Pierre Zoberman (dir.), Queer : Écritures de la différence ?- Autres temps, autres lieux, Paris, L'Harmattan, p. 187-217.

MASSAD, Joseph A. (2007), Desiring Arabs, Chicago, The University Chicago Press.

NCUBE, Gibson. (2014), «Repenser la construction transméditerranéenne de la sexualité "minoritaire": Rachid O., Abdellah Taïa et Eyet-Chékib Djaziri ", DIRE DIversités REcherches et terrains, no 5. p. 122-133.

RedouAnE, Najib. (2007), "Autobiographie transgressée chez Abdellah Taïa », dans Cristina Boidard (dir.), L'Autobiographie dans l'espace francophone, Cadiz, Chiclana, p. 103-130.

TAÏA, Abdellah. (2009), «L'homosexualité expliquée à ma mère », TelQuel, $n^{\circ} 367$, $<$ http://www.asymptotejournal.com/nonfiction/abdellahtaia-homosexuality-explained-to-my-mother/french/>.

—. (2010), Le Jour du Roi, Paris, Seuil.

-. (2011), "Lettre à Mohamed Bouazizi», Polka Magazine, no 12 , p. 52-63.

—. (2012), Infidèles, Paris, Seuil. 
-. (2012), "A boy to be sacrificed », New York Times, 24 mars, $<$ http://www.nytimes.com/2012/03/25/opinion/sunday/a -boy-to-be-sacrificed.html? $\mathrm{r}=0>$.

- . (2014), Arabs are no longer afraid. Texts on a revolution underway, Los Angeles, Semiotext(e).

Whitaker, Brian. (2006), Unspeakable Love: Gay and Lesbian Life in the Middle East, Londres, Saqi Books.

-. (2001), «Homosexuality on Trial in Egypt», The Guardian, 19 novembre.

<http://www.theguardian.com/world/2001/nov/19/world dispatch.brianwhitaker>.

ZEKRI, Khalid. (2008), «Littérature marocaine et transgression de l'hétéronormativité ", dans Pierre Zoberman (dir.), Queer : Écritures de la différence ?- Autres temps, autres lieux, Paris, L'Harmattan, p. 163-186.

\section{Résumé}

Cet article s'intéresse à l'évolution du rapport qu'entretient Abdellah Taïa avec les foules issues de mouvements populaires tel que le Printemps arabe. Alors que sa marginalité en tant qu'homosexuel et immigré paraît $a$ priori un obstacle à son engagement politique, elle finit par le conduire à innover et à envisager, à travers son écriture, les actions collectives comme garantissant le respect des particularités de chacun. Encore sceptique quant à la possibilité d'un mouvement contestataire uni dans Le Jour du Roi (2010), Taïa montre, à la suite du Printemps arabe, une volonté de passer d'une position d'observateur externe à une position plus participative au sein des foules dans Infidèles (2013). 


\begin{abstract}
This article examines the evolution of Abdellah Taïa's relations with crowds from popular movements such as the Arab Spring. If his marginality as a homosexual and an immigrant a priori seems an obstacle to his political engagement, such a position ends up leading him to innovate and consider, through his writing, collective actions guaranteeing the respect of everybody's singularities. Despite his skepticism about the possibility for a united protest movement in Le Jour $d u$ Roi (2010), Taïa shows, following the Arab Spring, a will to move from a position of external observer to a more participative position among the crowd through his novel Infidèles (2013).
\end{abstract}

